\title{
Caveolin-1 is a prognostic marker and suppresses the proliferation of breast cancer
}

\author{
Liping Ren ${ }^{1,2 \#}$, Peijuan Zhou ${ }^{3 \#}$, Huajia Wu ${ }^{1,2}$, Yuqi Liang ${ }^{1,2}$, Rui Xu ${ }^{1,2}$, Hai Lu ${ }^{1,2}$, Qianjun Chen ${ }^{1,2}$ \\ ${ }^{1}$ Department of Breast Disease, The Second Affiliated Hospital of Guangzhou University of Chinese Medicine, Guangzhou, China; ${ }^{2}$ Department \\ of Breast Disease, The Second Clinical College of Guangzhou University of Chinese Medicine, Guangzhou, China; ${ }^{3}$ Department of Traditional \\ Chinese Medicine, Renji Hospital, School of Medicine, Shanghai Jiaotong University, Shanghai, China \\ Contributions: (I) Conception and design: Q Chen, H Lu; (II) Administrative support: Q Chen; (III) Provision of study materials or patients: Y Liang; \\ (IV) Collection and assembly of data: $\mathrm{H} \mathrm{Wu}$; (V) Data analysis and interpretation: H Wu; (VI) Manuscript writing: All authors; (VII) Final approval \\ of manuscript: All authors. \\ \#These authors contributed equally to this work. \\ Correspondence to: Qianjun Chen, MD. Department of Breast Disease, The Second Affiliated Hospital of Guangzhou University of Chinese Medicine, \\ No. 55, West Ring Road, Guangzhou 510282, China. Email: cqj55@163.com; Hai Lu, MD. Department of Breast Disease, The Second Affiliated \\ Hospital of Guangzhou University of Chinese Medicine, No. 55, West Ring Road, Guangzhou 510282, China. Email: luhai@gzucm.edu.cn.
}

\begin{abstract}
Background: To explore the role of caveolin-1 (Cav-1) in breast cancer (BC).
Methods: Cav-1 expression data were downloaded from the Tumor Immune Estimation Resource (TIMER) and Gene Expression Omnibus (GEO) databases. We compared the expression of Cav-1 in different tumor tissues and between BC tissues and normal tissues (NTs), as well as the differences between different clinical traits. Kaplan-Meier survival analysis and univariate and multivariate Cox regression analyses were used to determine whether Cav-1 serves as a prognostic factor. The correlations of Cav-1 expression with the immune microenvironment and infiltrating immune cells were also analyzed. Quantitative polymerase chain reaction (qPCR) was used to detect Cav-1 mRNA expression in the MCF-7, SKB-R3, MDB-MB-231, and SUM-159 cell lines. LV-Cav-1-RNAi was transfected into MCF-7 and MDB-MB-231 cells, and the MTT assay was used to detect cell proliferation. Subsequently, MDB-MB-231 cells carrying the Cav-1-RNAi gene were used to determine the effects of Cav-1 knockdown on tumor growth in vivo using a severe combined immunodeficiency (SCID) model.
\end{abstract}

Results: Cav-1 was enriched in most solid tumors, and its expression was lower in BC tissues than in NT. Cav-1 expression was shown to be related to patients' clinical outcomes. Cav-1 was expressed in the MCF-7, SKB-R3, MDB-MB-231, and SUM-159 cell lines. The MTT assay revealed that the proliferative ability of MDB-MB-231 and MCF-7 cells was accelerated. The tumor volume of SCID mice administered with LVCav-1-RNAi cells was increased.

Conclusions: These results suggest that Cav-1 may serve as a suppressor in the development of BC.

Keywords: Breast cancer (BC); caveolin-1 (Cav-1); prognostic marker; tumor suppressor

Submitted Jun 17, 2021. Accepted for publication Aug 19, 2021.

doi: $10.21037 /$ tcr-21-1139

View this article at: https://dx.doi.org/10.21037/tcr-21-1139

\section{Introduction}

Breast cancer (BC) has become the most significant malignant tumor threatening women's lives (1). Over the past few decades, the model of $\mathrm{BC}$ treatment has changed dramatically, and the death rate among patients with $\mathrm{BC}$ has decreased significantly. However, the incidence of this disease is increasing year by year (2). Furthermore, the mechanism of $\mathrm{BC}$ development is still not fully understood.

Caveolin-1 (Cav-1) is an intrinsic membrane protein that can participate in tumor cell growth processes (3), including 
Table 1 Patients' information in the GSE1456 dataset

\begin{tabular}{lcc}
\hline Information & Cav-1 high $(\mathrm{n}=73)$ & Cav-1 low $(\mathrm{n}=74)$ \\
\hline Grade, $\mathrm{n}(\%)$ & & $10(13.5)$ \\
G1 & $18(24.7)$ & $21(28.4)$ \\
G2 & $37(50.7)$ & $43(58.1)$ \\
G3 & $18(24.7)$ & $55(74.3)$ \\
DSS, $\mathrm{n}(\%)$ & $65(89.0)$ & $19(25.7)$ \\
Alive & $8(11.0)$ & $51(68.9)$ \\
Dead & & $23(31.1)$ \\
OS, $\mathrm{n}(\%)$ & $61(83.6)$ & $12(16.4)$ \\
Alive & & \\
Dead & & \\
\hline
\end{tabular}

Cav-1, caveolin-1; DSS, disease-specific survival; OS, overall survival.

signal transduction and tumor progression. Cav-1 has both cancer-promoting (4) and anti-cancer effects (5). Cav-1 is highly expressed in tumor fibroblasts and secretes various cytokines or transcription factors involved in tumorigenesis and development (6,7). However, the gene encoding Cav1 is located primarily in the tumor suppressor region (7-9). Therefore, many scholars believe that $\mathrm{Cav}-1$ has a significant tumor-suppressive effect (10). Cav-1 is expressed at high or low levels in $\mathrm{BC}$, though literature reports are inconsistent. Therefore, there is no consensus as to whether Cav-1 is a tumor suppressor or oncogenic gene in BC (11-14).

Here, we analyzed the expression of the Cav-1 gene in $\mathrm{BC}$ by using large sample data from the Tumor Immune Estimation Resource (TIMER) and Gene Expression Omnibus (GEO) databases, and clarified the role of Cav-1 in the development of $\mathrm{BC}$ by performing in vivo and in vitro experiments using $\mathrm{BC}$ cell lines. We present the following article in accordance with the MDAR reporting checklist (available at https://dx.doi.org/10.21037/tcr-21-1139).

\section{Methods}

\section{Cav-1 expression level}

Cav-1 gene expression levels in different tumors were obtained from the TIMER database (https://cistrome. shinyapps.io/timer/) (15). BC and normal breast tissue gene expression chips were obtained from the GEO database. Expression data of $147 \mathrm{BC}$ tissues were obtained from the GSE1456 dataset (platform: GPL96). The clinical information is shown in Table 1. Normal breast tissue data were obtained from another dataset, GSE9574 (platform: GPL96), which included 14 matched normal breast tissues. The two datasets were merged, and the sva (16) package was used to eliminate batch effects.

\section{Survival analysis}

Survival analysis was used to calculate the overall survival (OS) and disease-specific survival (DSS) of patients with BC. Univariate and multivariate Cox regression analyses were used to determine whether Cav-1 expression could serve as an independent prognostic factor. The "SurvivalROC" package was used to generate receiver operating characteristic (ROC) curves and to calculate the area under the ROC curve (AUC) values.

\section{Gene set enrichment analysis (GSEA)}

GSEA was performed using GSEA web version (https:// www.gsea-msigdb.org/gsea/index.jsp). The c2.cp.kegg. v7.2.symbols.gmt gene set was selected to observe enriched pathways of the Cav-1 gene. The screening criteria were false discovery rate (FDR) $<0.25$ and an absolute value of enrichment score $(\mathrm{ES})>0.5$.

\section{Immune microenvironment analysis}

The ESTIMATE R package was used to calculate the immune cell and stromal cell scores in the immune 
microenvironment based on the expression matrix. Following the median expression value of the Cav-1 gene, there was a statistical difference in the scores of immune cells and stromal cells.

\section{Immune infiltration analysis}

The ESs of 28 immune cells were evaluated in each tumor sample using the single-sample GSEA (ssGSEA) method in the GSVA $\mathrm{R}$ package. We calculated the correlation between immune cells and the Cav-1 gene, and evaluated the relationship between the Cav-1 gene and immune cells.

\section{Cell culture}

The human BC cell lines MCF-1 and MDA-MB-231 were obtained from the American Type Culture Collection (ATCC, Manassas, VA, USA). The cells were cultivated in Dulbecco's modified Eagle medium (DMEM; SigmaAldrich, D777) with 10\% fetal bovine serum (Gibco Life Technologies, Lofer, Austria, 10099141) and 1\% penicillin and streptomycin (Sigma-Aldrich, V900929) at $37{ }^{\circ} \mathrm{C}$ in a $5 \% \mathrm{CO}_{2}$ environment.

\section{Reverse transcription-polymerase chain reaction (PCR)}

Total RNA was extracted from MCF-7, SKB-R3, MDA-MB-231, and SUM-159 cells using TRIzol, and reverse-transcribed to cDNA following the manufacturer's protocol (TAKARA, DRR041B). Realtime PCR was then used to detect the expression of Cav-1 (3'-GCAGAACCAGAAGGGACACACAG-5'; 5'-ACACGGCTGATGCACTGAACTC-3') in MCF7, SKB-R3, MDA-MB-231, and SUM-159 cells, with GAPDH used as an internal control.

\section{The construction of lentiviruses containing short bairpin RNA (shRNA) against Cav-1}

The shRNA sequence (CCTTGTTCCTGAAACAATT) was designed and used to target the Cav-1 gene. A scrambled sequence (TTCTCCGAACGTGTCACGT) was set as a negative control construct. Single-stranded DNA with the interfering sequence was inserted into the lentivirus green fluorescent protein (GFP) vector GV115 (Jikai, Shanghai, China) using T4 DNA ligase (Promega, SU3604). The ligated vector was transfected into competent TOP10 Escherichia coli. (Zhujiang Hospital).

\section{Lentivirus transfection}

The vectors with correct sequences were transfected into 293 cells. After 48 hours, the virus in the supernatant was concentrated to the target volume by centrifugation. $\mathrm{BC}$ MCF-7 and MDA-MB-231 cells were seeded into 6-well plates and transfected with concentrated lentivirus in the presence of polybrene (Sigma-Aldrich, TR1003) according to the manufacturer's instructions. When the GFP expression exceeded $80 \%$ in each group, cells were selected using puromycin (Sigma, P3388, $0.5 \mu \mathrm{g} / \mathrm{mL}$ ).

\section{Examination of knockdown efficiency by quantitative real- time PCR}

Total RNA was extracted from transfected MCF-7 and MDA-MB-231 cells using a TRIzol extraction kit (Pufei Biotechnology Co., Ltd., Shanghai, China) and reversetranscribed into cDNA using Promega M-MLV reverse transcriptase, according to the manufacturers' protocols. PCR amplification was conducted with SYBR Master Mixture (TAKARA, DRR041B). The following reaction conditions were used: $95{ }^{\circ} \mathrm{C}$ for 5 seconds, followed by 45 cycles at $95^{\circ} \mathrm{C}$ for 5 seconds, and $60{ }^{\circ} \mathrm{C}$ for 30 seconds. The $2^{-\Delta \Delta}$ method was applied to analyze the data.

\section{Western blotting analysis}

After lentivirus transfection, cells were lysed in radioimmunoprecipitation assay buffer (Sigma Aldrich), and the protein concentration was measured with the bicinchoninic acid assay (Sigma). Protein lysates $(10 \mu \mathrm{g})$ were resolved by sodium dodecyl sulfate-polyacrylamide gel electrophoresis, transferred onto a polyvinylidene difluoride membrane (Millipore), and immunoblotted with antibodies including Cav-1 antibody (Abcam, ab32577) and GAPDH antibody (Abcam, ab9485) at $4{ }^{\circ} \mathrm{C}$ overnight. The membrane was then incubated with goat anti-rabbit IgG (Abcam, ab6721) for 2 hours at room temperature after 3 washes with tris-buffered saline with $0.05 \%$ Tween-20. The signals were visualized using the ECL advance reagent (Tagno Science \& Technology, Shanghai, China) and quantified using ImageLab software.

\section{MTT assay}

Cells were seeded into 96-well plates (5,000 cells/well) and incubated at $37{ }^{\circ} \mathrm{C}$ in a $5 \% \mathrm{CO}_{2}$ environment for an additional 1, 2, 3, 4, and 5 days. At each time point, after 
the addition of $5 \mathrm{mg} / \mathrm{mL} 3$-(4,5-dimethylthiazol-2-yl)-2,5diphenyltetrazolium bromide (MTT, $20 \mu \mathrm{L} /$ well), the cells were incubated for another 4 hours, and the supernatants were removed. Next, $100 \mu \mathrm{L}$ dimethyl sulfoxide (DMSO) was added to each well, and the cells were incubated for a further 10 minutes with constant shaking. The absorbance at $490 \mathrm{~nm}$ was measured using a spectrophotometric plate reader, and cell growth curves were plotted.

\section{Tumor formation in mice with severe combined immunodeficiency (SCID)}

The MDB-MB-231 cells infected with Cav-1-RNAiLentivirus vector and negative-control vector were resuspended in phosphate-buffered saline (PBS). Five-weekold female SCID mice ( $\mathrm{n}=8$ each group) were subcutaneously injected with $200 \mu \mathrm{L}$ of PBS containing $2 \times 10^{6}$ cells $/ \mathrm{mL}$ via the armpit. Tumor volume assessment started on the $5^{\text {th }}$ day after cell injection and was conducted twice a week.

\section{Ethical statement}

All procedures involving animals followed the ethical standards of the Animal Ethical Committee of the Second Affiliated Hospital of Guangzhou University of Chinese Medicine (No. 2020031) and were conducted according to the National Institutes of Health Guide for the Care and Use of Laboratory Animals. All animal experiments were performed in the Animal Experiment Center of Guangdong Academy of Chinese Medical Sciences. The study was conducted in accordance with the Declaration of Helsinki (as revised in 2013).

\section{Statistical analysis}

$\mathrm{R}$ version 3.6.3 was used for statistical analysis. Due to the uneven distribution of samples, the Wilcoxon signed-rank test was used to differentiate Cav-1 expression in normal tissues (NTs) and BC tissues, and the Kolmogorov-Smirnov test was used to analyze the difference in Cav-1 expression and clinical pathology. SPSS 20 was used to analyze the data with a pairwise $t$-test and analysis of variance, and $\mathrm{P}<0.05$ was considered statistically significant.

\section{Results}

\section{Cav-1 gene expression level}

First, we evaluated the expression level of Cav-1 in
BC. We observed low expression of the Cav-1 gene in $\mathrm{BC}$ tissues from the TIMER database and also found statistically significant Cav-1 expression in most solid tumors. Compared with NT, low expression of Cav-1 was found in bladder cancer, colon adenocarcinoma, kidney renal papillary cell carcinoma, lung adenocarcinoma, lung squamous cell carcinoma, prostate adenocarcinoma, stomach adenocarcinoma, thyroid cancer, and uterine corpus endometrial carcinoma. High expression of Cav-1 was found in cholangiocarcinoma, Head and Neck squamous cell carcinoma, chromophobe renal cell carcinoma, renal cell clear cell carcinoma, and hepatocellular carcinoma compared with NT (Figure 1A). Since the GSE1456 dataset contained only 147 BC tissues, to fully evaluate the expression levels of the Cav-1 gene, we obtained the expression data of 14 normal breast tissues from the GSE9574 dataset and integrated the two datasets. We found that Cav-1 expression was significantly lower in $\mathrm{BC}$ tissues than in NTs (Figure $1 B, \mathrm{P}<0.0001$ ). Cluster analysis showed that the $\mathrm{BC}$ samples and normal samples could be clustered into two categories (Figure 1C).

\section{Prognostic significance of the Cav-1 gene in BC}

We used the median expression value of the Cav-1 gene to observe the difference in OS and DSS between patients with high and low Cav-1 expression. Patients with low Cav1 expression had a lower OS rate than patients with high expression (Figure 2A, $\mathrm{P}=0.043$ ). However, comparable results were observed between the two groups of patients with respect to DSS (Figure $2 B, \mathrm{P}=0.021$ ). We observed that the lower the expression of $C a v-1$, the lower the tumor grade and the higher the degree of malignancy (Figure 2C). ROC analysis showed that the AUCs for OS and DSS were 0.71 (Figure 2D) and 0.604 (Figure 2E), respectively. Next, we compared the relationship between Cav-1 and tumor grade. The univariate Cox regression and Cox regression analysis results showed $\mathrm{P}=0.012$ (Figure $3 \mathrm{~A}$ ) and $\mathrm{P}=0.057$ for $\mathrm{OS}$ (Figure $3 B$ ) respectively, and also $\mathrm{P}=0.003$ (Figure $3 C$ ) and $\mathrm{P}=0.033$ for DSS (Figure $3 D$, Table 2) respectively.

\section{Cav-1 gene patbway enrichment}

To further determine the biological functions and pathways in which Cav-1 is involved, GSEA was conducted. The results showed that $C a v-1$ inhibited the DNA replication and cell cycle pathways (Figure 4A,4B). 


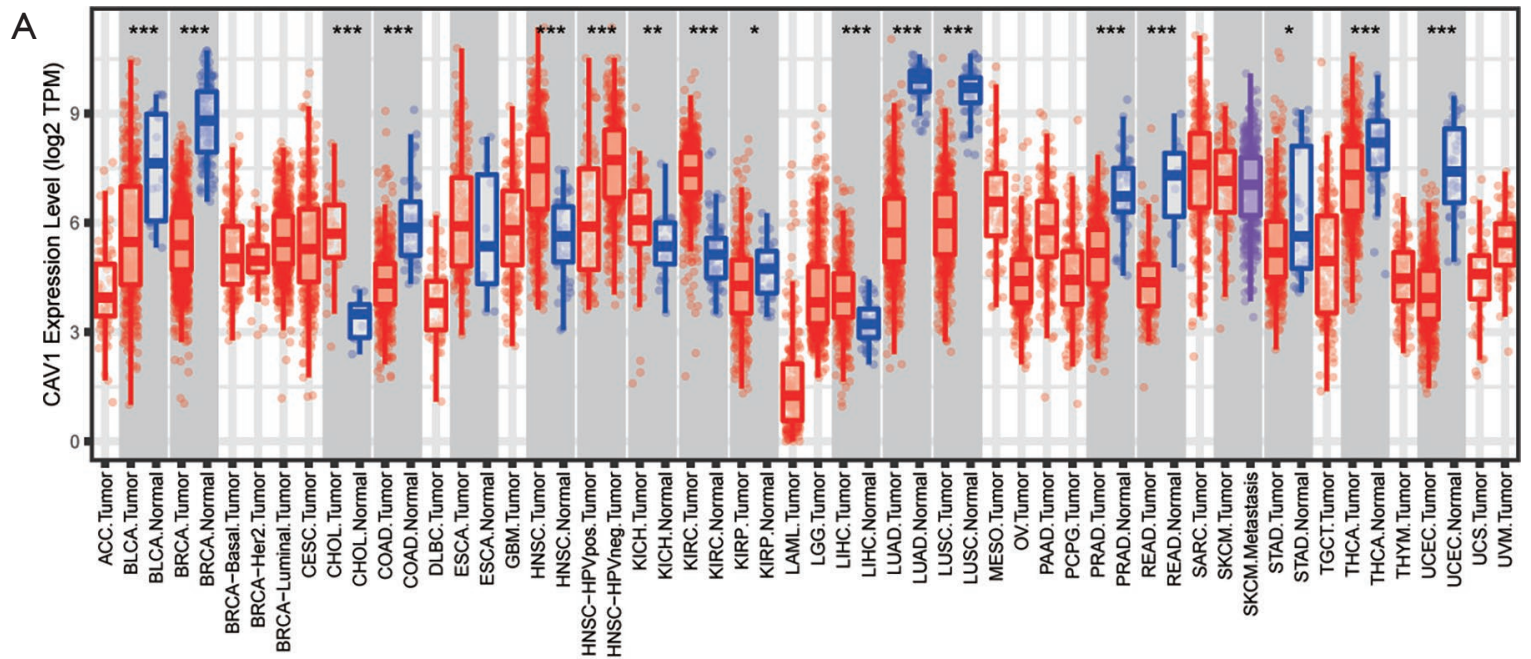

B

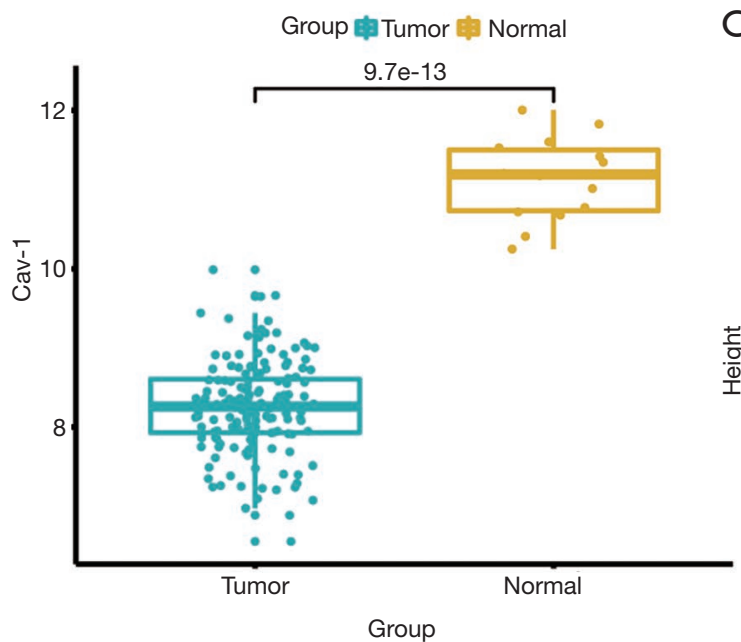

C Cluster dendrogram

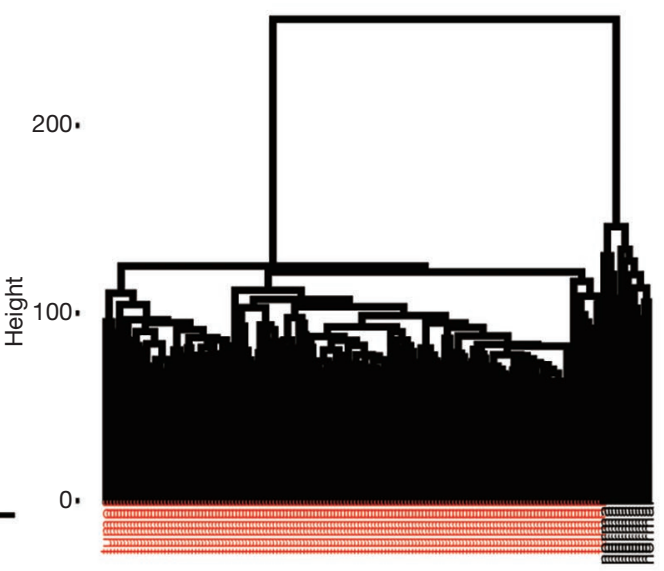

Figure 1 Cav-1 expression levels in human tumors and BC. (A) The expression levels of the Cav-1 gene in different tumors in the TIMER database (*, $\mathrm{P}<0.05$; **, $\mathrm{P}<0.01$; **, $\mathrm{P}<0.001$ ). (B) The expression levels of the Cav-1 gene in the integrated GSE1456 and GSE9574 datasets. (C) Cluster diagram of samples in the GSE1456 and GSE9574 datasets. Cav-1, caveolin-1; BC, breast cancer; TIMER, Tumor Immune Estimation Resource.

\section{Correlation between Cav-1 expression and the tumor immune microenvironment}

Correlation analysis of Cav-1 expression and immune cell score, stromal cell score, estimated score, and tumor purity in BC showed that Cav-1 expression was positively correlated with the stromal and estimated scores but negatively correlated with tumor purity (Figure $5 A$ ). The correlations of Cav-1 with the stromal score (Figure 5B) and the estimated score (Figure 5C) were statistically significant $(\mathrm{P}<0.001)$. Also, the matrix score of the high Cav-1 expression group was found to be significantly higher than that of the low expression group $(\mathrm{P}<0.0001$, Figure 5D). However, there was no significant difference in immune scores between the groups (Figure 5E). With respect to tumor grade, the higher the grade, the lower the stromal score (Figure 5F).

\section{Correlations between Cav-1 and infiltrating immune cells}

Using the ssGSEA method, we obtained the scores of 28 immune cells in BC tissues. Heat map analysis showed that most of the immune cell scores were greater than 0 (Figure 6A). As shown in the correlation diagram, Cav- 
A

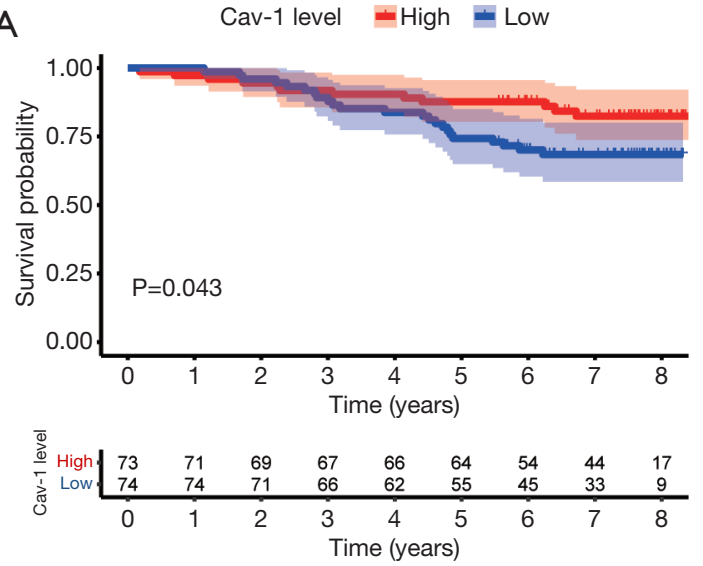

C

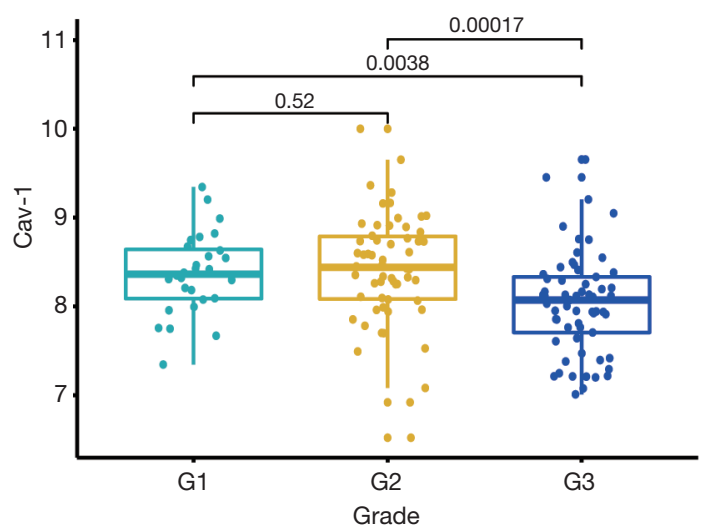

D

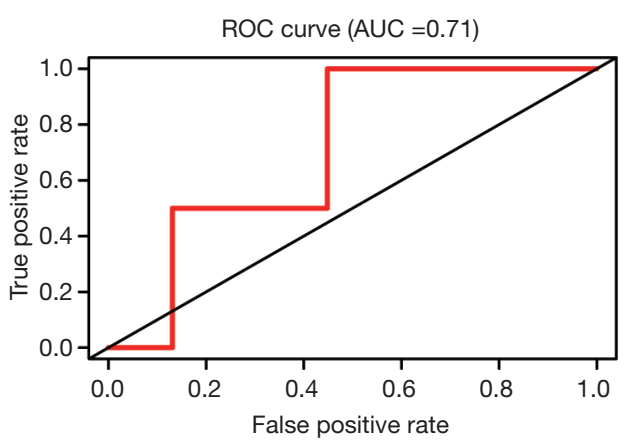

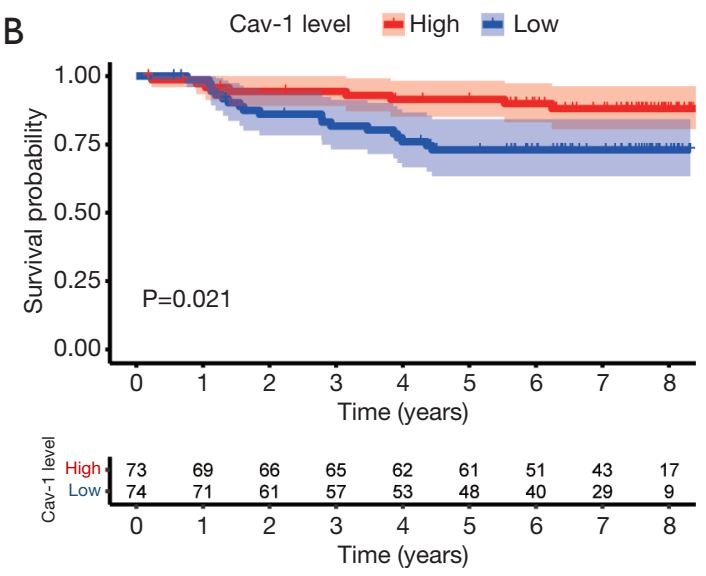

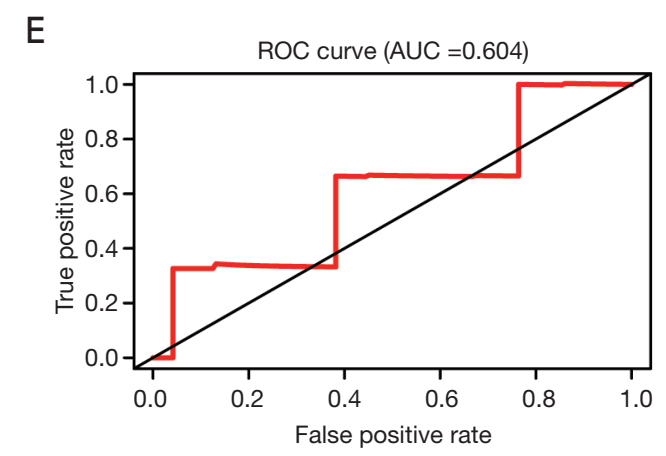

$\mathrm{E}$

Figure 2 Clinical significance of Cav-1. (A) Correlation of Cav-1 with OS in the GSE1456 dataset. (B) Correlation of Cav-1 with DSS in the GSE1456 dataset. (C) The relationship between Cav-1 and tumor grade. (D) The ROC curve of Cav-1 expression for predicting OS. (E) The ROC curve of Cav-1 expression for predicting DSS. Cav-1, caveolin-1; OS, overall survival; DSS, disease-specific survival; ROC, receiver operating characteristic; $\mathrm{AUC}$, area under the ROC curve.

1 was positively correlated with most immune cells (Figure 6A,6B). The immune cells significantly positively correlated with $C a v-1$ were plasmacytoid dendritic cells, neutrophils, mast cells, immature B cells, eosinophils, CD56 bright natural killer (NK) cells, effector memory
CD4 T cells, NK cells, type $1 \mathrm{~T}$ helper cells, $\mathrm{T}$ follicular helper cells, effector memory CD8 $\mathrm{T}$ cells, regulatory $\mathrm{T}$ cells, central memory CD4 T cells, and gammadelta $\mathrm{T}$ cells. The immune cells significantly negatively correlated with Cav-1 were CD4 T cells, CD56 dim NK cells, and 


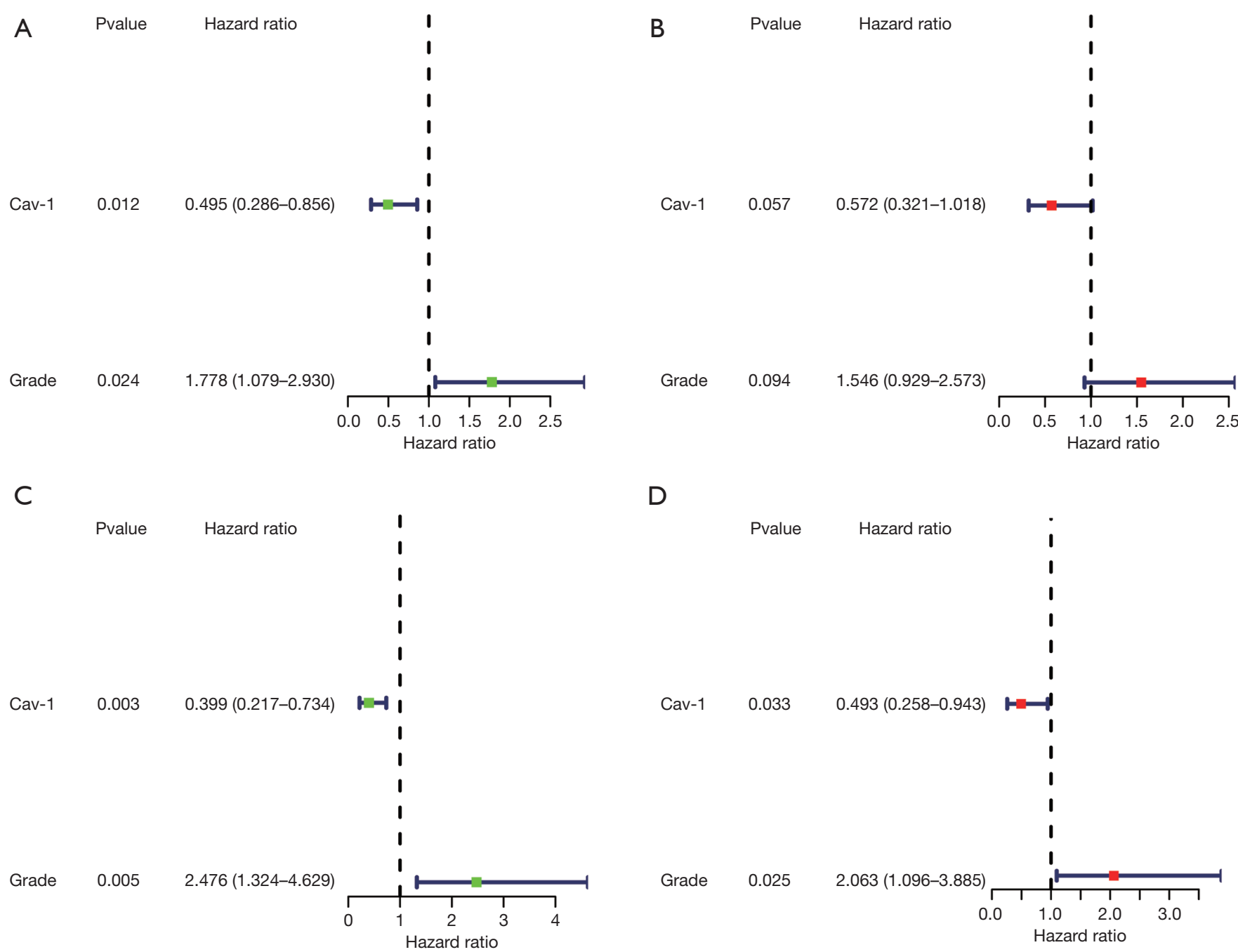

Figure 3 Univariate regression analysis and multivariate regression analysis of the relationship between Cav-1 expression and OS (A,B) and DSS (C,D). Cav-1, caveolin-1; OS, overall survival; DSS, disease-specific survival.

Table 2 Univariate analysis of OS and DSS in patients with BC

\begin{tabular}{|c|c|c|c|c|c|c|c|c|}
\hline Parameters & \multicolumn{4}{|c|}{ OS } & \multicolumn{4}{|c|}{ DSS } \\
\hline Cav-1 & 0.495 & 0.286 & 0.856 & 0.012 & 0.399 & 0.217 & 0.734 & 0.003 \\
\hline Grade & 1.778 & 1.079 & 2.930 & 0.024 & 2.476 & 1.324 & 4.629 & 0.005 \\
\hline
\end{tabular}

OS, overall survival; DSS, disease-specific survival; BC, breast cancer; Cav-1, caveolin-1.

dendritic cells (Figure 6C). Among the immune cells with a significant correlation with $C a v-1$, those associated with high expression of Cav-1 were plasmacytoid dendritic cells (Figure 6D), neutrophils (Figure 6E), mast cells (Figure 6F), immature B cells (Figure 6G), eosinophils (Figure 6H), and
CD56 bright NK cells. The immune score of NK cells (Figure 6I) was higher than that of the low expression group. However, for activated dendritic cells (Figure 67) and CT4T cells (Figure $6 \mathrm{~K}$ ), the immune score of the high expression group was lower than that of the low expression group. 

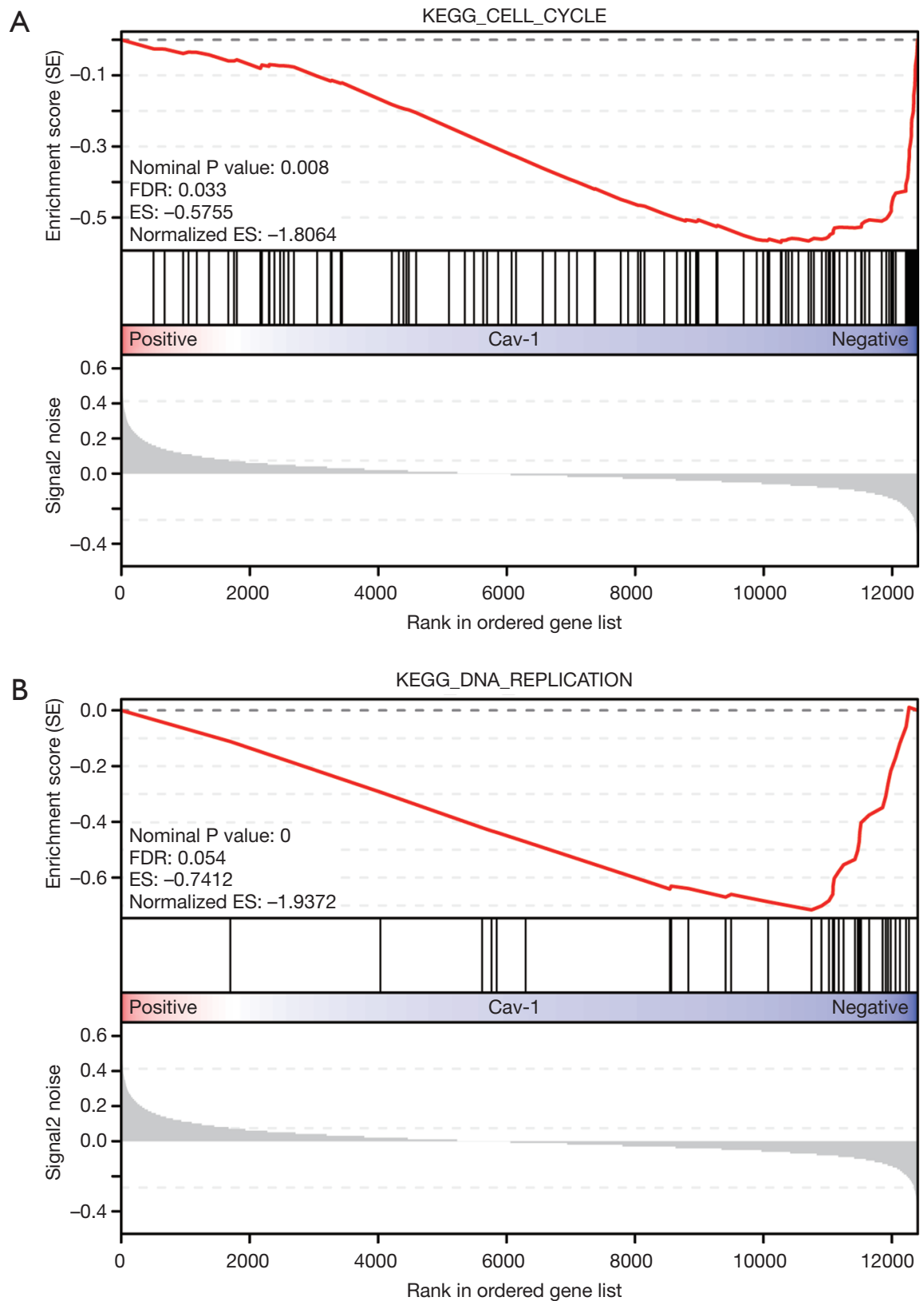

Figure 4 Kyoto Encyclopedia of Genes and Genomes pathway enrichment analysis of the Cav-1 gene. (A) Cav-1 is enriched in the cell cycle, and (B) DNA replication pathways. Cav-1, caveolin-1; FDR, false discovery rate; ES, enrichment score.

\section{Silencing of Cav-1 stimulated BC cell growth}

The results of PCR and western blotting showed that Cav1 was expressed in $\mathrm{BC}$ cell lines, with the highest expression detected in triple-negative BC cells (Figure $7 A, 7 B$ ). Also, the proliferation of transfected MCF-7 and MDA-MB-231 cells was detected by the MTT assay. As shown in Figure 7, for both MCF-7 (Figure 7C,7D) and MDA-MB-231 cells (Figure $7 E, 7 F)$, the cell proliferation rate in the shCav-1 group was faster than that in the shCtrl group $(\mathrm{P}<0.05)$. These results demonstrated that the knockdown of endogenous Cav-1 by RNAi stimulated the proliferation of BC cells. 

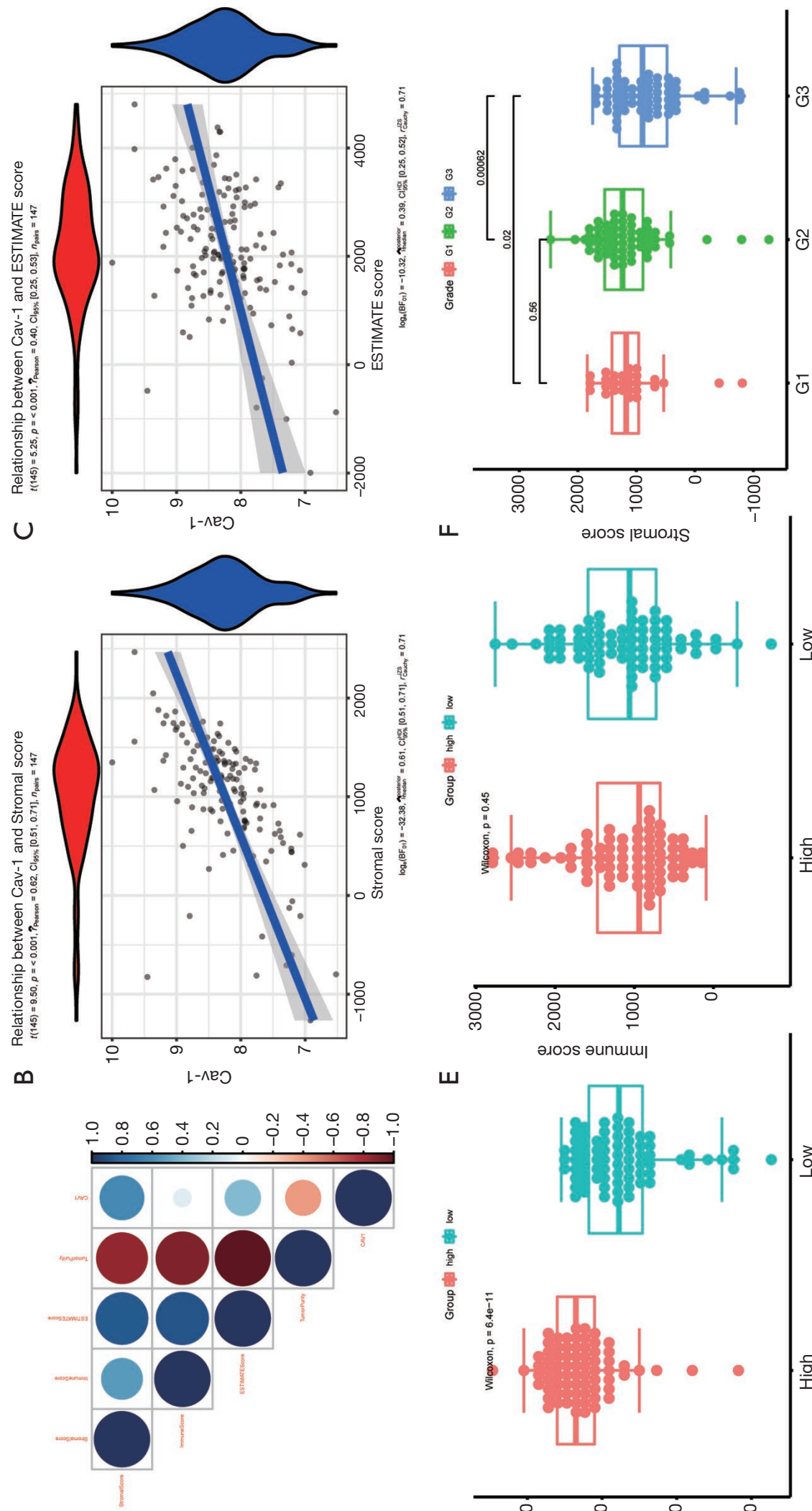

$\varangle$

ш

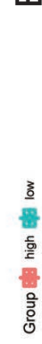

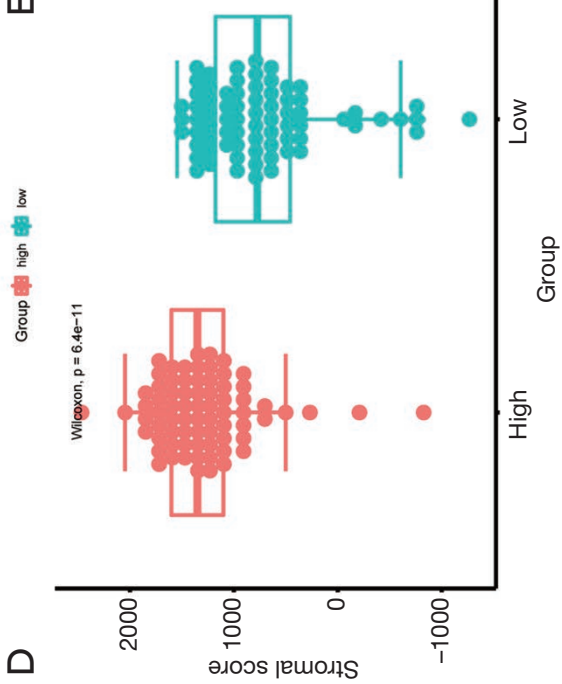

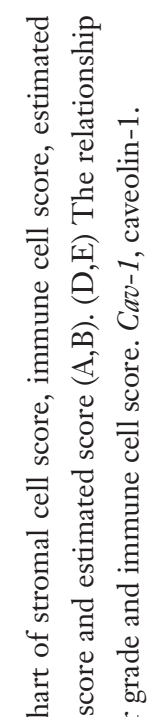

चี

热

ن

¿

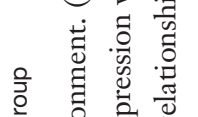

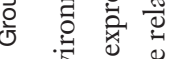

ปี

每整无

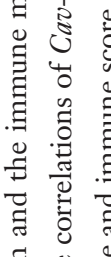

苛害

ठิ

๑े

㟧

गิ

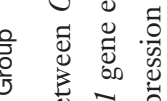

ป d

击

总氩苛

ह 己

ज官兵

的寻

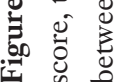




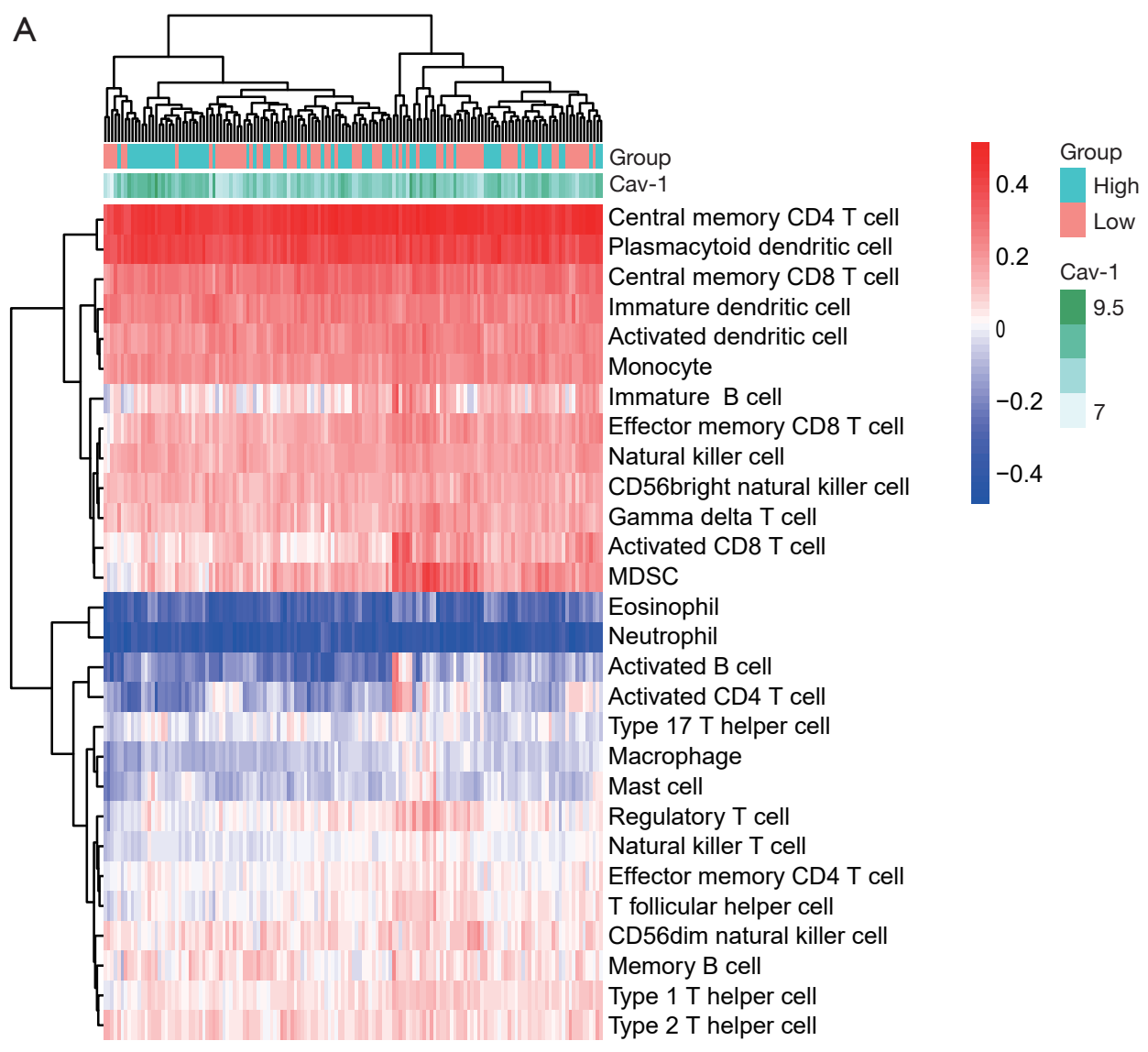

B

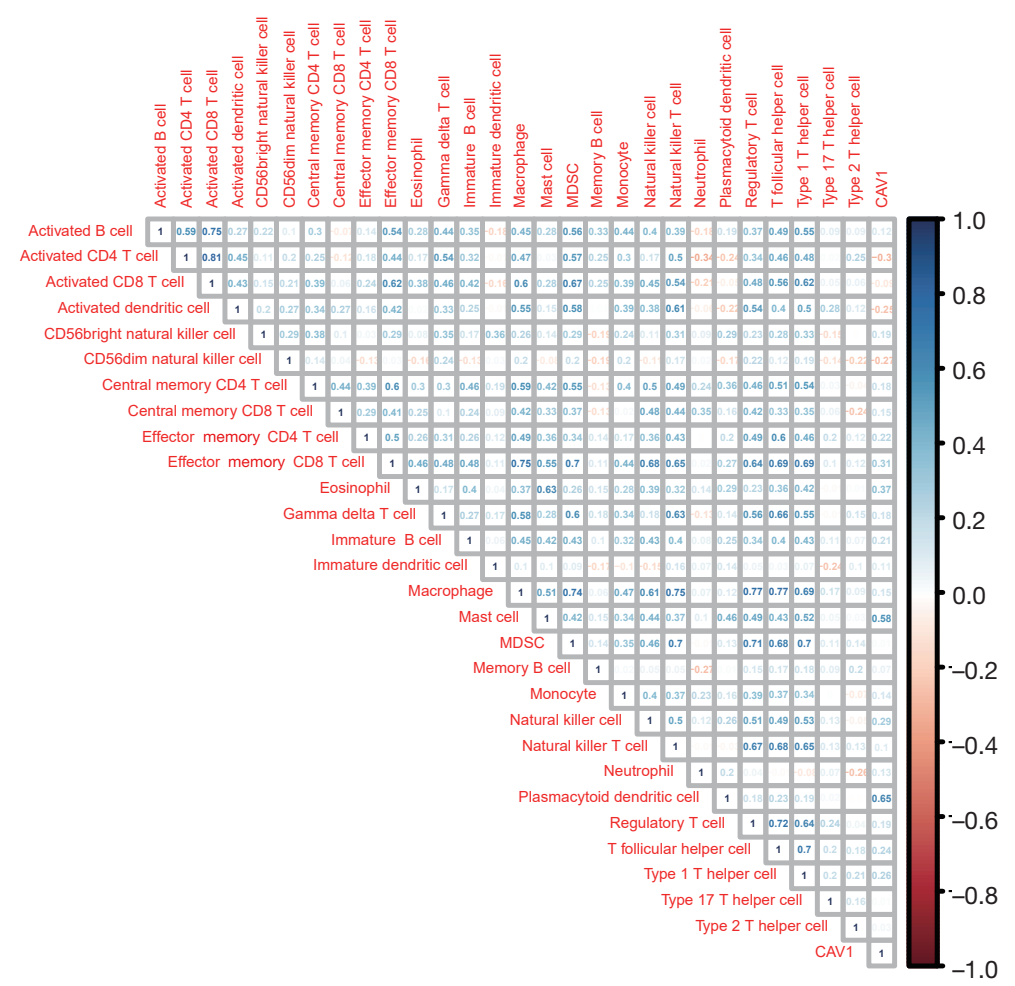




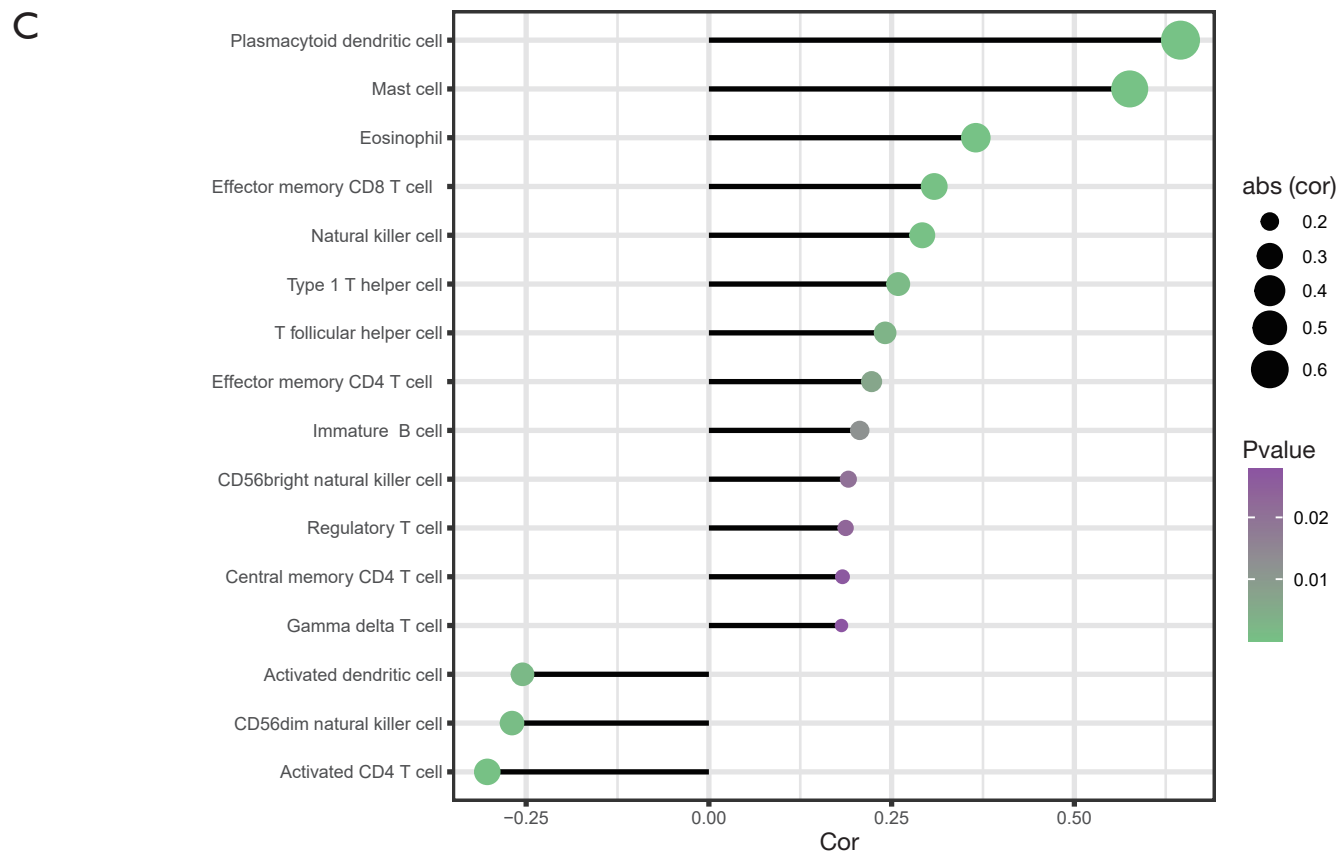

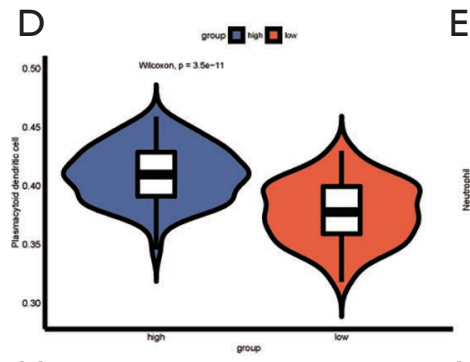

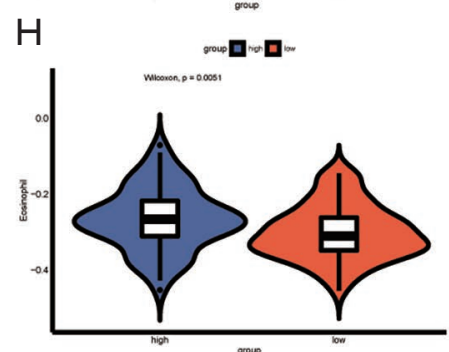

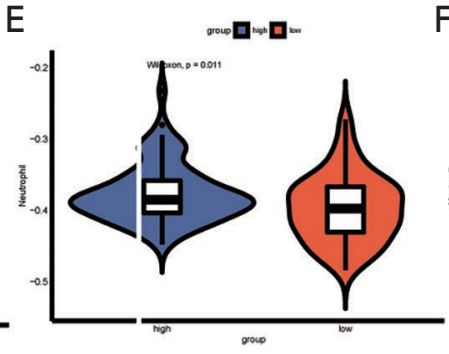
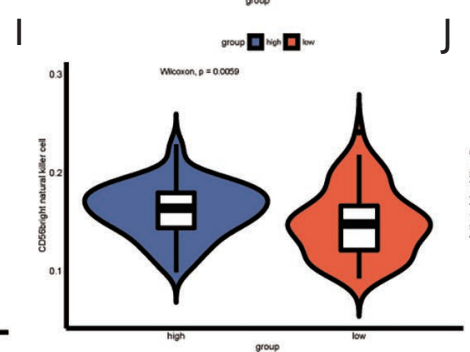
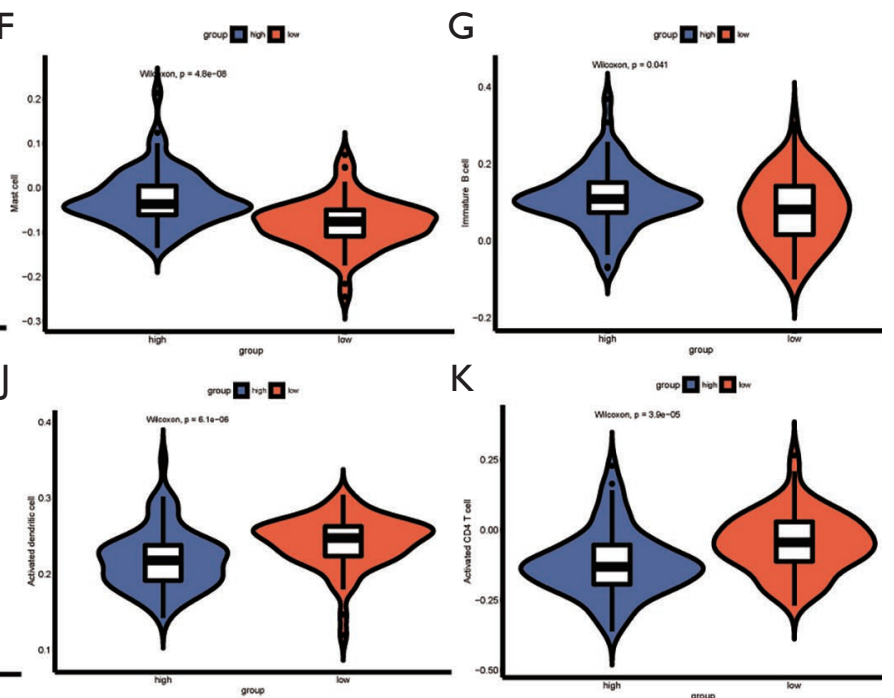

Figure 6 The relationship between Cav-1 and immune cells was analyzed through ssGSEA. (A) Heat map of immune cell fractions. (B) Correlation diagram of immune cell score and Cav-1 gene expression. (C) Immune cells with significant correlations with Cav-1 gene expression (selection criteria: $\mathrm{P}<0.05$ ). (D-K) The relationships between Cav-1 gene expression and plasmacytoid dendritic cells (D), neutrophils (E), mast cells (F), immature B cells $(\mathrm{G})$, eosinophils (H), CD56 bright NK cells (I), activated dendritic cells (J), and activated CD4 T cells (K). Cav-1, caveolin-1; ssGSEA, single-sample gene set enrichment analysis; NK, natural killer.

\section{Cav-1 knockdown in MDB-MB-231 cells increased tumor growth in vivo}

To investigate whether Cav-1 acts as a suppressor of BC in vivo, MDB-MB-231 cells from two groups (negative control and Cav-1-RNAi) were subcutaneously injected into SCID mice. The tumor volumes of the Cav-1-RNAi group were significantly greater than those of the negative control group from day 15 to 21 after implantation (Figure $7 G$ ). 
A
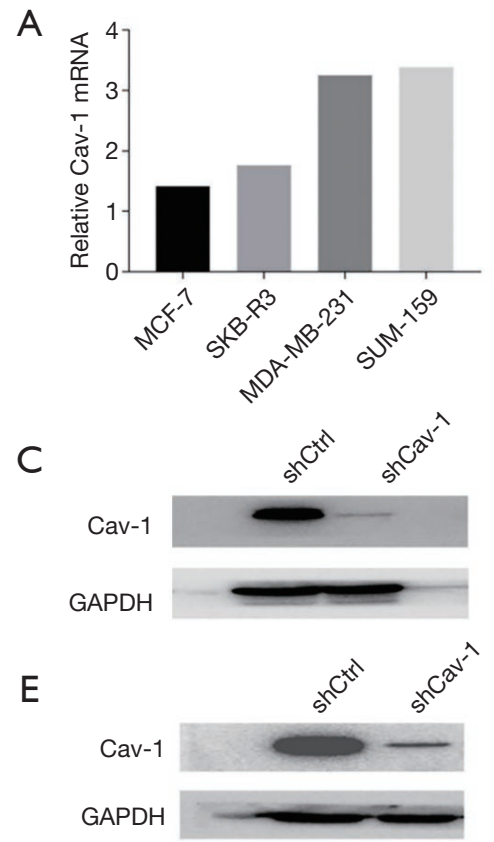

G

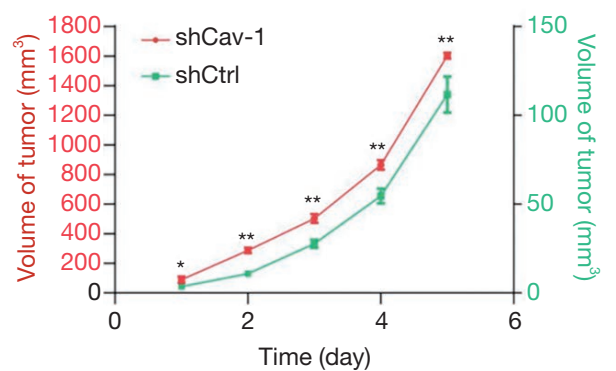

B

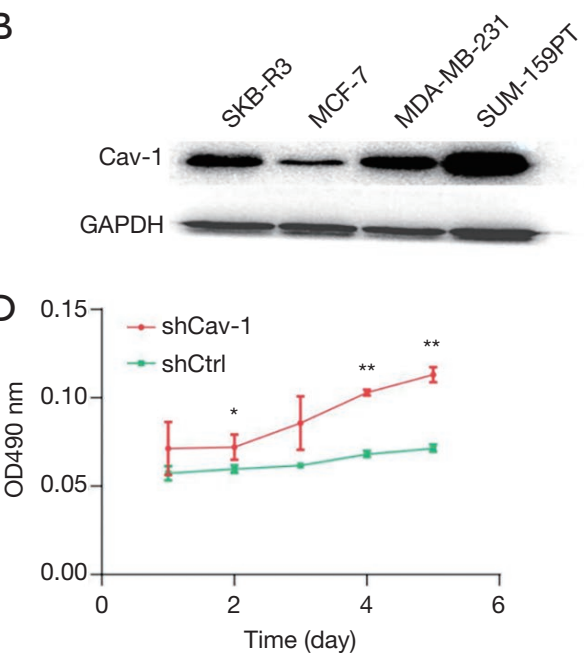

$\mathrm{F}$

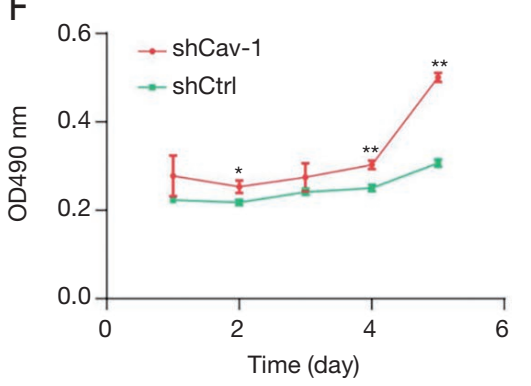

Figure 7 Cav-1 expression in BC cells. The expression of Cav-1 was detected in BC cell lines by PCR (A) and western blotting (B). Cav-1 was expressed in BC cell lines and was highly expressed in triple-negative BC cells. Knockdown of Cav-1 expression in MCF-7 cells (C) and MDB-MB-231 cells (E). The proliferation of MCF-7 cells (D) and MDB-MB-231 (F) cells was accelerated (shCtrl: control group; shCav-1: Cav-1 knockdown group). (G) The tumor volume increased after Cav-1 knockdown [(data were analyzed for percent apoptotic cells and represented as mean $\pm \operatorname{SEM}\left({ }^{*}, \mathrm{P}<0.05\right.$; $\left.{ }^{* *}, \mathrm{P}<0.01\right)$ ]. Cav-1, caveolin-1; BC, breast cancer; PCR, polymerase chain reaction.

\section{Discussion}

Recently, a study confirmed that high levels of Cav-1 in the stromal tissue surrounding a breast tumor are strongly associated with reduced metastasis and improved survival (17). However, no study has verified whether the expression of Cav-1 can be considered a new surface marker in BC. In our study, we found that Cav-1 is expressed at low levels in BC and can act as a tumor suppressor to inhibit BC progression. Our results also showed that low Cav-1 expression is a strong predictor of tumor recurrence.

Cav-1 is a member of the caveolin gene family along with Cav-2 and Cav-3 (18). Cav-1 is expressed in a wide variety of tissues, whereas Cav-3 is only expressed in muscle tissue (19). Cav-2 is co-expressed with Cav-1, which it requires for stabilization and plasma membrane localization (20). The association between $\mathrm{Cav}-1$ and cancer progression has already been established (21). Clinically, a loss of stromal Cav-1 is associated with a poor outcome, especially for patients with prostate cancer or BC (22-24). One potential mechanism of this is the upregulation of transforming growth factor-beta (TGFB) expression in Cav-1-deficient fibroblasts, which was observed to foster epithelial Cav-1 expression, promoting epithelial-to-mesenchymal transition $(25,26)$.

Cav-1 may also serve as a powerful predictive biomarker in BC, as stromal loss of Cav-1 expression and increased 
epithelial Cav-1 expression have been associated with a larger tumor size, a higher rate of nodal involvement, and an increased number of involved lymph nodes (8,24,27-29). For patients with BC who have low Cav-1 expression, the 10 -year survival rate is $43 \%$, compared with $91 \%$ in patients with high Cav-1 expression (24,30). Loss of Cav-1 has prognostic value for patients with particularly aggressive subtypes of BC, such as triple-negative and basal-like BC. The 5-year survival rate is $75 \%$ for patients with high expression of Cav-1, compared with $9 \%$ for patients with low expression of Cav-1 (31).

\section{Conclusions}

In summary, our findings show that low Cav-1 expression is associated with more aggressive disease in patients with BC. Thus, Cav-1 may be considered as a tumor suppressor gene in BC.

\section{Acknowledgments}

The authors are very grateful to the data providers of the study.

Funding: This study was supported by the National Natural Science Foundation of China (No. 81974571), Guangdong Natural Science Foundation (No. 2017A030313719), and China Postdoctoral Science Foundation (No. 2020M682683).

\section{Footnote}

Reporting Checklist: The authors have completed the MDAR reporting checklist. Available at https://dx.doi. org/10.21037/tcr-21-1139

Data Sharing Statement: Available at https://dx.doi. org/10.21037/tcr-21-1139

Conflicts of Interest: All authors have completed the ICMJE uniform disclosure form (available at https://dx.doi. org/10.21037/tcr-21-1139). The authors have no conflicts of interest to declare.

Ethical Statement: The authors are accountable for all aspects of the work in ensuring that questions related to the accuracy or integrity of any part of the work are appropriately investigated and resolved. All procedures involving animals followed the ethical standards of the
Animal Ethical Committee of the Second Affiliated Hospital of Guangzhou University of Chinese Medicine (No. 2020031) and were conducted according to the National Institutes of Health Guide for the Care and Use of Laboratory Animals. All animal experiments were performed in the Animal Experiment Center of Guangdong Academy of Chinese Medical Sciences. The study was conducted in accordance with the Declaration of Helsinki (as revised in 2013).

Open Access Statement: This is an Open Access article distributed in accordance with the Creative Commons Attribution-NonCommercial-NoDerivs 4.0 International License (CC BY-NC-ND 4.0), which permits the noncommercial replication and distribution of the article with the strict proviso that no changes or edits are made and the original work is properly cited (including links to both the formal publication through the relevant DOI and the license). See: https://creativecommons.org/licenses/by-nc-nd/4.0/.

\section{References}

1. Siegel RL, Miller KD, Jemal A. Cancer statistics, 2020. CA Cancer J Clin 2020;70:7-30.

2. Harbeck N, Penault-Llorca F, Cortes J, et al. Breast cancer. Nat Rev Dis Primers 2019;5:66.

3. Mohamed FEA, Khalil EZI, Toni NDM. Caveolin-1 expression together with VEGF can be a predictor for lung metastasis and poor prognosis in osteosarcoma. Pathol Oncol Res 2020;26:1787-95. Erratum in: Pathol Oncol Res 2020;(26):2013-4.

4. Galbiati F, Volonte D, Engelman JA, et al. Targeted downregulation of caveolin-1 is sufficient to drive cell transformation and hyperactivate the $\mathrm{p} 42 / 44$ MAP kinase cascade. EMBO J 1998;17:6633-48.

5. Park J, Bae E, Lee C, et al. RNA interference-directed caveolin-1 knockdown sensitizes SN12CPM6 cells to doxorubicin-induced apoptosis and reduces lung metastasis. Tumour Biol 2010;31:643-50.

6. Parton RG. Caveolae: structure, function, and relationship to disease. Annu Rev Cell Dev Biol 2018;34:111-36.

7. Simón L, Campos A, Leyton L, et al. Caveolin-1 function at the plasma membrane and in intracellular compartments in cancer. Cancer Metastasis Rev 2020;39:435-53.

8. El-Gendi SM, Mostafa MF, El-Gendi AM. Stromal caveolin-1 expression in breast carcinoma. Correlation with early tumor recurrence and clinical outcome. Pathol Oncol Res 2012;18:459-69. 
9. Kruglikov IL, Scherer PE. Caveolin-1 as a possible target in the treatment for acne. Exp Dermatol 2020;29:177-83.

10. Ketteler J, Klein D. Caveolin-1, cancer and therapy resistance. Int J Cancer 2018;143:2092-104.

11. Patani N, Martin LA, Reis-Filho JS, et al. The role of caveolin-1 in human breast cancer. Breast Cancer Res Treat 2012;131:1-15.

12. Sotgia F, Rui H, Bonuccelli G, et al. Caveolin-1, mammary stem cells, and estrogen-dependent breast cancers. Cancer Res 2006;66:10647-51.

13. Shi Y, Tan SH, $\mathrm{Ng} \mathrm{S}$, et al. Critical role of CAV1/ caveolin-1 in cell stress responses in human breast cancer cells via modulation of lysosomal function and autophagy. Autophagy 2015;11:769-84.

14. Cift T, Begum AM, Aslan Cetin B, et al. Serum caveolin-1 levels in patients with preeclampsia. J Matern Fetal Neonatal Med 2020;33:712-7.

15. Li T, Fu J, Zeng Z, et al. TIMER2.0 for analysis of tumor-infiltrating immune cells. Nucleic Acids Res 2020;48:W509-14.

16. Chakraborty S, Datta S, Datta S. Surrogate variable analysis using partial least squares (SVA-PLS) in gene expression studies. Bioinformatics 2012;28:799-806.

17. Shan-Wei W, Kan-Lun X, Shu-Qin R, et al. Overexpression of caveolin- 1 in cancer-associated fibroblasts predicts good outcome in breast cancer. Breast Care (Basel) 2012;7:477-83.

18. Nwosu ZC, Ebert MP, Dooley S, et al. Caveolin-1 in the regulation of cell metabolism: a cancer perspective. Mol Cancer 2016;15:71.

19. Galbiati F, Engelman JA, Volonte D, et al. Caveolin-3 null mice show a loss of caveolae, changes in the microdomain distribution of the dystrophin-glycoprotein complex, and t-tubule abnormalities. J Biol Chem 2001;276:21425-33.

20. Parolini I, Sargiacomo M, Galbiati F, et al. Expression of caveolin-1 is required for the transport of caveolin-2 to the plasma membrane. Retention of caveolin-2 at the level of the golgi complex. J Biol Chem 1999;274:25718-25.

21. Chen D, Che G. Value of caveolin-1 in cancer progression and prognosis: Emphasis on cancer-associated fibroblasts, human cancer cells and mechanism of caveolin-1 expression (Review). Oncol Lett 2014;8:1409-21.

22. Ayala G, Morello M, Frolov A, et al. Loss of caveolin-1 in prostate cancer stroma correlates with reduced relapse-free survival and is functionally relevant to tumour progression. J Pathol 2013;231:77-87.

23. Kibria G, Hatakeyama H, Harashima H. Cancer multidrug resistance: mechanisms involved and strategies for circumvention using a drug delivery system. Arch Pharm Res 2014;37:4-15.

24. Witkiewicz AK, Dasgupta A, Sotgia F, et al. An absence of stromal caveolin-1 expression predicts early tumor recurrence and poor clinical outcome in human breast cancers. Am J Pathol 2009;174:2023-34.

25. Panic A, Ketteler J, Reis H, et al. Progression-related loss of stromal Caveolin 1 levels fosters the growth of human PC3 xenografts and mediates radiation resistance. Sci Rep 2017;7:41138.

26. Gottlieb-Abraham E, Shvartsman DE, Donaldson JC, et al. Src-mediated caveolin-1 phosphorylation affects the targeting of active Src to specific membrane sites. Mol Biol Cell 2013;24:3881-95.

27. Mercier I, Casimiro MC, Wang C, et al. Human breast cancer-associated fibroblasts (CAFs) show caveolin-1 downregulation and $\mathrm{RB}$ tumor suppressor functional inactivation: Implications for the response to hormonal therapy. Cancer Biol Ther 2008;7:1212-25.

28. Eliyatkin N, Aktas S, Diniz G, et al. Expression of stromal caveolin- 1 may be a predictor for aggressive behaviour of breast cancer. Pathol Oncol Res 2018;24:59-65.

29. Finak G, Bertos N, Pepin F, et al. Stromal gene expression predicts clinical outcome in breast cancer. Nat Med 2008;14:518-27.

30. Sloan EK, Ciocca DR, Pouliot N, et al. Stromal cell expression of caveolin-1 predicts outcome in breast cancer. Am J Pathol 2009;174:2035-43.

31. Witkiewicz AK, Dasgupta A, Sammons S, et al. Loss of stromal caveolin-1 expression predicts poor clinical outcome in triple negative and basal-like breast cancers. Cancer Biol Ther 2010;10:135-43.

(English Language Editor: C. Betlazar-Maseh)
Cite this article as: Ren $\mathrm{L}$, Zhou $\mathrm{P}, \mathrm{Wu} \mathrm{H}$, Liang $\mathrm{Y}$, $\mathrm{Xu} \mathrm{R}, \mathrm{Lu} \mathrm{H}$, Chen Q. Caveolin-1 is a prognostic marker and suppresses the proliferation of breast cancer. Transl Cancer Res 2021;10(8):3797-3810. doi: 10.21037/tcr-21-1139 